\title{
Selective visual attention modulates the direct tilt aftereffect
}

\author{
MICHAEL J. SPIVEY and MARC J. SPIRN \\ Cornell University, Ithaca, New York
}

\begin{abstract}
One's being able to allocate attention to particular regions or properties of the visual field is fundamental to visual information processing. Visual attention determines what input is carefully analyzed and what input is more or less ignored. But at what stage of the visual system is this process evident? We describe three experiments that demonstrate an effect of voluntary spatial attention and voluntary object-based attention on an orientation illusion (the tilt aftereffect) that is believed to take place in primary visual cortex. This finding, in which selective visual attention influences adaptation to visual orientation information, contributes to mounting evidence for a view of visual perception in which mutual interaction takes place between high-level and low-level subsystems.
\end{abstract}

Theories of attention have varied widely in where they place the influence of voluntary attention in the architecture of the human perceptual/cognitive system. Classical theories of attention propose either an "early filter" that blocks or attenuates unattended sensory information before cognitive processing (Broadbent, 1958; Treisman, 1964) or a "late filter" that discards information from the unattended channel only after automatic semantic analysis (Deutsch \& Deutsch, 1963; Norman, 1976). In the past couple of decades, the latter account, in which certain perceptual processes are encapsulated from higher level processes, has received considerable support in the area of vision research (e.g., Livingstone \& Hubel, 1988; Marr, 1982; Pylyshyn, 1999; Shallice, 1988; Zeki, 1993).

More recently, however, this modular feedforward view of the visual system has been undergoing significant revision, due in part to an increased understanding of the importance of feedback projections and dynamic receptive fields (e.g., Churchland, Ramachandran, \& Sejnowski, 1994; Douglas, Koch, Mahowald, Martin, \& Suarez, 1995; Gilbert, 1993; Lamme, Super, \& Spekreijse, 1998). Moreover, recent neuroimaging and electrophysiology studies have shown specifically that voluntary attention can indeed modulate activity in primary visual cortex (Artim \& Bridgeman, 1989; Brefczynski \& DeYoe, 1999; Gandhi, Heeger, \& Boynton, 1998; Haenny \& Schiller, 1988; Motter, 1993; Roelfsema, Lamme, \& Spekreijse,

We are grateful to Jeremy Wolfe, Mary Hayhoe, Virginia de Sa, Leslie Cameron, Joel Lachter, the Visual Attention Reading Group at the University of Rochester, and two anonymous reviewers for helpful comments and to Tania Pasternak for the use of her equipment. This work was supported by an NSF Graduate Research Fellowship and a Sloan Foundation Fellowship in Neuroscience to M. J. Spivey, and a student grant from the Department of Neurobiology and Behavior at Cornell University to M. J. Spirn. M. J. Spirn is now at the Robert Wood Johnson Medical School. Address correspondence to M. J. Spivey. Department of Psychology, Cornell University, Ithaca, NY 14853 (e-mail: spivey@cornell.edu).
1998; Sengpiel \& Hubener, 1999). Within the domain of human visual psychophysics, contributions to this rethinking of visual perception have come in the form of demonstrations of attention modulating putatively automatic perceptual processes, such as the motion aftereffect (Chaudhuri, 1990; Lankheet \& Verstraten, 1995; Rees, Frith, \& Lavie, 1997) and figural aftereffect (Shulman, 1992; Yeh, Chen, DeValois, \& DeValois, 1996). By the same logic, the present study takes the direct tilt aftereffect (TAE), which is typically understood to be an automatic adaptation process in primary visual cortex, and shows modulation of its magnitude via selective visual attention.

The direct TAE is demonstrated in Figure 1. Following prolonged viewing of a slightly tilted grating, observers perceive a vertical grating (in the same region of the visual field) as being tilted in the opposite direction (Gibson \& Radner, 1937; Morant \& Harris, 1965; Muir \& Over, 1970). Psychophysical (Blakemore, Carpenter, \& Georgeson, 1970; Carpenter \& Blakemore, 1973; Magnussen \& Kurtenbach, 1980; Wenderoth, van der Zwan, \& Johnstone, 1989), electrophysiological (Blakemore \& Tobin, 1972; Morrone, Burr, \& Maffei, 1982; Nelson \& Frost, 1978), neurochemical (Sillito, 1979), neuroanatomical (Gilbert \& Weisel, 1983), and computational (Bednar \& Miikkulainen, 1997; Spivey-Knowlton, 1993) results suggest that such angular repulsion is due to lateral inhibition between orientation-selective cells in primary visual (striate) cortex. For example, Blakemore and Tobin recorded activity from complex cells in areas 17 and 18 of the cat and found that when a cell's preferred orientation stimulus is surrounded by an annulus grating with a similar orientation, the cell's firing rate is reduced almost to resting level.

Lateral inhibition between orientation-selective cells in the primary visual cortex may be useful for sharpening orientation-selectivity bandwidths (Blakemore et al., 1970; Sillito, 1979). As a side effect, it may cause TAEs by residual mutual repulsion of stimulus-evoked activity pat- 

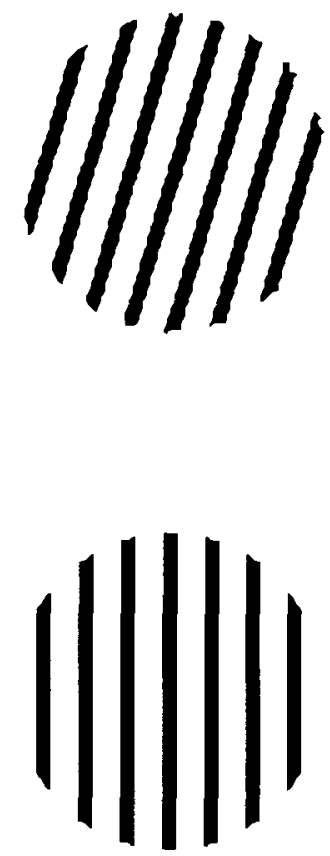

Figure 1. The tilt aftereffect (TAE). Stare at the center of the upper grating for 1 min, and then look at the lower grating. This lower grating, though actually vertical, should appear tilted slightly counterclockwise from vertical.

terns. For example, assume a cell tuned to $75^{\circ}\left(15^{\circ}\right.$ clockwise from vertical) inhibits cells tuned to $90^{\circ}$ and $60^{\circ}$ and all orientations in between. After such inhibition has taken place, when a vertical stimulus is then presented, the cells tuned to $90^{\circ}, 85^{\circ}$, and $80^{\circ}$ will have weak responses due to the inhibition. However, cells tuned to $95^{\circ}$ and $100^{\circ}$ (slightly counterclockwise from vertical) will not be affected by the inhibition. Thus, the distribution of activity across the cells that usually respond to the vertical stimulus will be slightly skewed toward counterclockwise from vertical. Can this phenomenon, which presumably takes place in primary visual cortex (Wenderoth et al., 1989), be modulated by voluntary attention?

In the first two experiments presented here, the participants selectively attended to one identically oriented stimulus over another, while maintaining fixation equidistant from the two stimuli. Following adaptation, the TAE was measured for the attended region as opposed to the unattended region. In the third experiment, the participants attended to one color of a symmetric green and red plaid, after which the TAE was tested in that same region. In these experiments, we tested whether selectively allocating visual attention to a region of the visual field (Experiments 1 and 2) or to a property of the inducing stimulus (Experiment 3 ) would affect orientation processing and its concomitant lateral inhibition in primary visual cortex.

\section{EXPERIMENT 1}

The participants were instructed to fixate a point equidistant from two adaptation gratings (both set at $15^{\circ}$ from vertical) and to selectively attend to one of the gratings for $1 \mathrm{~min}$. Although the participants found that it required effort, it was not difficult to maintain fixation on the central dot while attending to one of the two stimuli (see Figure 2). If the processing of orientation information (and its concomitant lateral inhibition) for both gratings was unaffected by the observer's selective allocation of spatial attention, the magnitude of the TAE should be equal for the attended and unattended regions.

\section{Method}

Participants. Eight adults, all naive to the hypothesis, participated in this experiment. Six were experienced psychophysical observers. Half of the participants adapted to $15^{\circ}$ clockwise from vertical and half adapted to $15^{\circ}$ counterclockwise from vertical. Each participant was run for a total of eight trials, or 16 settings of apparent vertical.

Stimuli. Gratings were displayed on a Hewlett-Packard 1332A CRT by an Innisfree Picasso Image Synthesizer. Black cardboard was placed over the display, which revealed a small central dot for eye fixation and two circular apertures whose centers were $5.8^{\circ}$ of arc left and right of fixation. The circular apertures had radii of $5^{\circ}$ of arc. Square wave gratings of 0.5 cycles $/ \mathrm{deg}, 16.6 \%$ luminance contrast, and a mean luminance of $7.6 \mathrm{~cd} / \mathrm{m}^{2}$ were presented in the circular apertures. Orientation was controlled by a rotation switch in front of the participant.

Procedure. Room lights were turned off during trials. The participants were instructed to fixate the center dot and attend to one of the gratings for $1 \mathrm{~min}$. The experimenter punctuated this adaptation period with reminders to fixate center and attend to the appropriate grating. The grating attended to on a trial (left or right), the grating first tested (attended or unattended), and the starting ori-
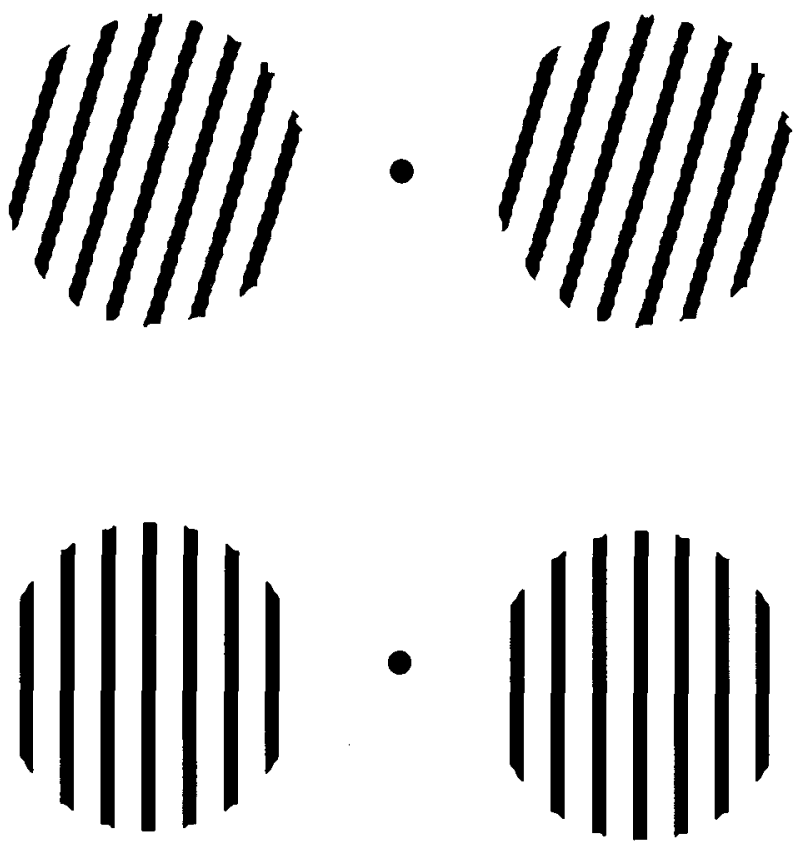

Figure 2. Participants fixated a central dot and attended to one of the gratings for $1 \mathrm{~min}$ (upper row), and then, during central fixation, set one of the gratings to apparent vertical. After selectively attended adaptation in the upper row, the reader may fixate the central dot in the lower row of gratings and observe that the grating on the unattended side appears closer to true vertical. Hence, the TAE is slightly larger in the attended adaptation field. 
entation for the test grating ( $5^{\circ}$ clockwise or counterclockwise) were all counterbalanced within subjects, and order was randomized between subjects. After adaptation, a panel occluded one of the gratings, orientation was set to $5^{\circ}$ from vertical, and the participant then (while maintaining fixation of the dot) adjusted the orientation to apparent vertical. Immediately after this first setting, the occluders were reversed to reveal the other grating, orientation was set back to $5^{\circ}$ from vertical, and the participant adjusted this grating to apparent vertical (while fixating the dot). Two-minute breaks intervened between each trial. An independent measure of attention allocation as per instruction during adaptation, such as a secondary detection task (Shulman, 1992), was not performed. However, since the comparison of interest is between identical stimulus conditions, the only manipulation being the attentional instruction, any difference in TAE magnitude can only be attributed to an influence of selective attention.

\section{Results and Discussion}

Six of the 8 participants showed similar effects of spatial attention's increasing the TAE. Averaged across all participants, the TAE for the attended grating $\left(1.44^{\circ}\right)$ was $20 \%$ larger than for the unattended grating $\left(1.2^{\circ}\right)[t(7)=$ $3.23, p<.02]$. Since the second setting made by participants might reflect weakened effects due to decay of the TAE, a $t$ test was also conducted on the participants' first settings alone. In this analysis, the attended grating showed a TAE $\left(1.69^{\circ}\right)$ that was $39 \%$ larger than for the unattended grating $\left(1.22^{\circ}\right)[t(7)=3.07, p<.02]$. See Figure 3 .

Although it is possible that, during adaptation, participants' fixations might have deviated somewhat toward the attended grating and away from the unattended grat- ing, this should not be responsible for the difference in TAE magnitudes. Deviated fixation would displace the retinotopic adaptation fields induced by the two gratings by an equal amount. During the testing phase, this equal displacement of the adaptation fields relative to the test gratings should decrease the TAEs for both gratings about equally.

Given these data, voluntary spatial attention appears to have influenced the putatively automatic processing of orientation information. Although this influence was somewhat mild (clearly less than $50 \%$ modulation), it was, nonetheless, present and statistically reliable. Accounts of orientation perception and the TAE that rule out attentional influences would have difficulty accounting for such a result.

\section{EXPERIMENT 2}

Although the results of Experiment 1 were initially compelling, there is still some concern that the participants might not have maintained central fixation during the adaptation and test phases. Moderate deviations in fixation could perhaps induce shifted adaption fields, with the attended grating's adaptation field being somewhat closer to the fovea than was the unattended grating's adaptation field. Adaptation fields closer to the fovea could perhaps induce a greater TAE than could more peripheral adaptation fields; however, this has not been systematically studied. It may be worth noting that a sim-

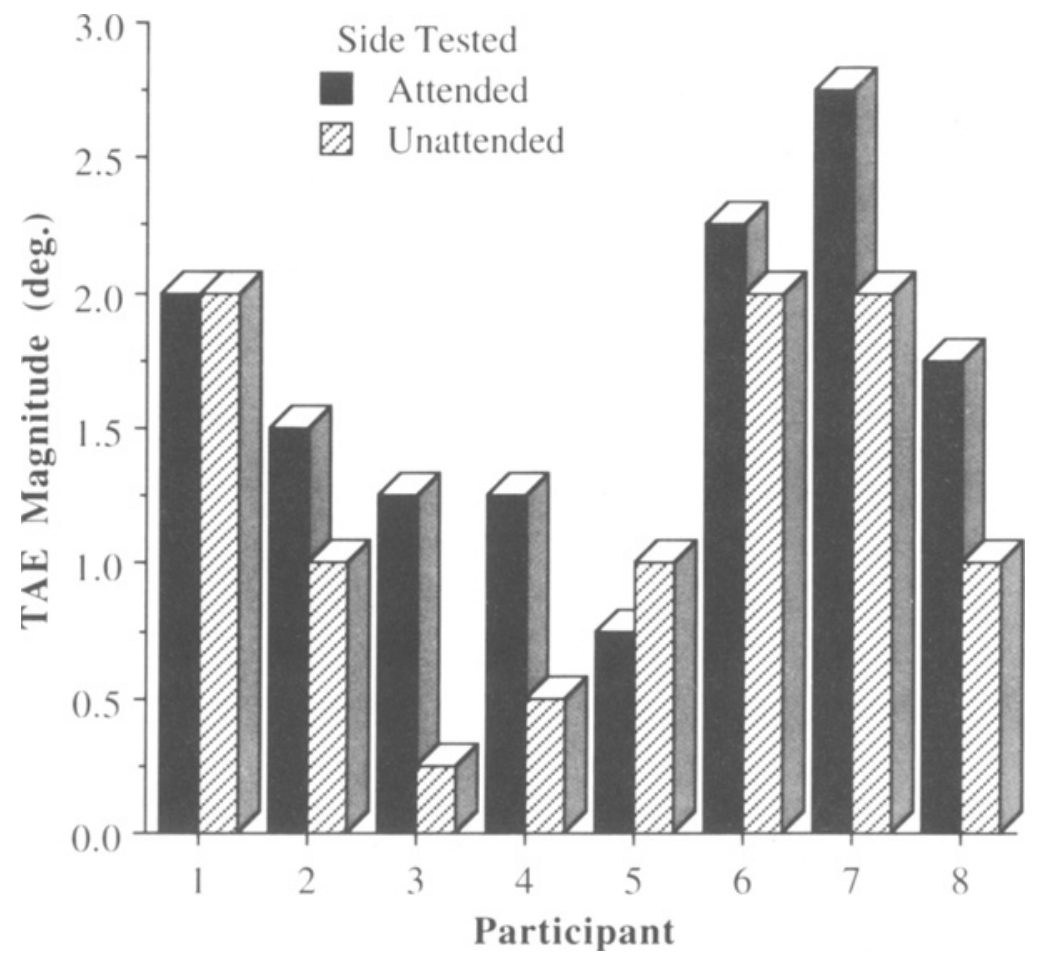

Figure 3. Experiment 1. The magnitude of TAE in the test grating when participants were tested in the attended adaptation field versus the unattended adaptation field of the same stimulus (data from first measurement only). 
ilar orientation illusion, the rod-and-frame effect, has a constant magnitude out to about $35^{\circ}$ visual angle (Ebenholtz, 1985). Nonetheless, any failures to maintain central fixation in the previous experiment would complicate interpretation of the data.

To address this concern, the basic experiment was repeated with central fixation monitored by an eyetracker, and trials in which fixation deviated from center were discarded. In this attempt to replicate an attentional modulation of the TAE, we chose to present brief flashes of the test gratings, because this produces increased magnitudes of the basic TAE (Wolfe, 1984), and to have participants report whether the test grating was clockwise or counterclockwise from vertical. With TAEs of potentially greater magnitude, we expected to have more "room" to observe the attentional modulation.

\section{Method}

Participants. Nine adults, all naive to the hypothesis, participated in this experiment. None of them were experienced psychophysical observers. All had normal or corrected-to-normal vision. Four of the participants adapted to $15^{\circ}$ clockwise from vertical and 5 adapted to $15^{\circ}$ counterclockwise from vertical. One participant, from the group that adapted to counterclockwise from vertical, was unable to finish the experiment; his data were not included. Each participant ran in two sessions consisting of 10 trials each.

Stimuli. Gratings were presented on a Macintosh 25-in. color monitor. Black cardboard was placed over the display, which revealed a large circle with a radius of $27.5^{\circ}$ of arc. This cardboard was used to reduce framing effects. For the adaptation phase, there were two circular gratings, each of which was centered at an eccentricity of $14.8^{\circ}$ of arc from the center fixation cross, and had radii of $6.1^{\circ}$ of arc. All grating stimuli were black and white bars with a spatial frequency of 0.5 cycles/deg and had $97 \%$ luminance contrast and a mean luminance of $2.4 \mathrm{~cd} / \mathrm{m}^{2}$. The center of fixation was a black cross subtending $1.1^{\circ}$ of visual arc. This center cross was surrounded by a square that subtended $3.4^{\circ}$ of visual arc. During the adaptation phase, the orientation of both gratings was set to either $15^{\circ}$ clockwise from vertical or $15^{\circ}$ counterclockwise from vertical.

During the test phase, one circular grating was presented for $50 \mathrm{msec}$ either on the left or on the right in the same location and size as was the previous adaptation grating (the central fixation stimulus remained on the screen). The test grating varied randomly among $0^{\circ}, 2^{\circ}, 4^{\circ}, 6^{\circ}$, and $8^{\circ}$ from vertical. If the adaptation stimulus was clockwise, the test stimulus was also clockwise; similarly, a counterclockwise adaptation stimulus had a counterclockwise test stimulus. During the time when the participant was choosing whether the test grating was clockwise or counterclockwise, only the center of fixation was present.

Procedure. Room lights were turned off during trials. The participants were instructed to fixate on the center cross and attend to one of the gratings for $55 \mathrm{sec}$. Forty seconds into each trial, the participant was reminded to maintain fixation on the center cross and to continue attending to the appropriate grating. Fixation of the central box was recorded by an ISCAN eyetracker mounted on top of a lightweight headband. The camera provided an infrared image of the left eye sampled at $60 \mathrm{~Hz}$. The center of the pupil and the corneal reflection were tracked to determine the direction of the eye relative to the head. A scene camera, yoked with the view of the tracked eye, provided an image of the participant's field of view. Gaze position (indicated by crosshairs) was superimposed over the scene camera image and recorded onto a $\mathrm{Hi} 8 \mathrm{VCR}$ with $30-\mathrm{Hz}$ frame-by-frame playback. Accuracy of the gaze position record was within $1^{\circ}$ visual angle. Deviation from the central fixation box invalidated the trial and the corresponding datum was excluded from analysis. In this way we could exclude cases of overt orienting and count only cases of covert (attentional) orienting.

During the first session, a participant adapting to counterclockwise from vertical gratings would attend to the right grating on each of the 10 trials. After attending to the right grating, the participant would be tested with either a left or a right test grating. The test gratings varied randomly between right and left and also among $0^{\circ}, 2^{\circ}, 4^{\circ}$, $6^{\circ}$, and $8^{\circ}$ counterclockwise from vertical. The second session was exactly the same except the participant was instructed to attend to the left grating instead of the right grating. For the participants adapting to clockwise from vertical gratings, the testing phase was identical to the testing phase of the participants adapting to counterclockwise except the test gratings were $0^{\circ}, 2^{\circ}, 4^{\circ}, 6^{\circ}$, and $8^{\circ}$ clockwise from vertical. Each test stimulus was presented $350 \mathrm{msec}$ after the end of the adaptation phase and lasted $50 \mathrm{msec}$. After being presented with the test stimulus, the participants were instructed to choose whether the test stimulus was clockwise or counterclockwise. The participant input the choice by choosing one of two keys on the computer, one that was designated counterclockwise and one that was designated clockwise. Between trials, a 40 -sec break interval allowed the participant to de-adapt.

\section{Results and Discussion}

When a participant perceived a clockwise test stimulus as being clockwise this was coded the same as when a participant perceived a counterclockwise stimulus as being counterclockwise (i.e., a veridical percept). Likewise, a clockwise stimulus perceived as being counterclockwise was coded the same as a counterclockwise stimulus perceived as being clockwise (i.e., a TAE). Although this was a rather coarse method of coding the responses, it provided a stringent and conservative measure of the approximate magnitude of TAE in these briefly flashed test stimuli. For example, when an $8^{\circ}$ clockwise test grating was perceived as $1^{\circ}$ clockwise it would be coded as a veridical percept, and when $a 6^{\circ}$ clockwise test grating was perceived as $1^{\circ}$ counterclockwise it would be coded as a TAE. Interpolation across these two hypothetical trials would accurately suggest that the magnitude of the TAE in this case was approximately $7^{\circ}$.

The participants in this task did occasionally fail to maintain central fixation. Trials in which the participants' fixation deviated even slightly from the central fixation box $(21 \%$ of the trials) were excluded from analysis. Rather than a constant deviated fixation off center, the vast majority of these deviations in fixation during adaptation comprised saccades to the attended grating and quick return saccades to the central fixation cross. Although there may still be some concern about minor deviations of fixation within the central fixation box, this control substantially reduced the likelihood that any observed effects were due to eye position instead of covert attention.

Although the raw data were binomial, the repeated measures design required that the data be averaged within a subject before being averaged across subjects. This re- 
sulted in an approximately normal distribution of the data before it was averaged across subjects. Therefore, the data were subjected to a $t$ test.

Overall, test gratings on the attended side produced more TAE responses $(75 \%)$ than test gratings on the unattended side did $(53 \%)[t(7)=2.57, p<.05]$. This effect was particularly apparent with the test gratings tilted at $2^{\circ}$ and $6^{\circ}$ (see Figure 4 ). Gratings tilted at $2^{\circ}$ produced significantly more TAEs on the attended side $(94 \%)$ than on the unattended side $(50 \%)[t(7)=2.37, p<.05]$. Likewise, gratings tilted at $6^{\circ}$ produced more TAEs on the attended side $(67 \%)$ than on the unattended side $(25 \%)$ $[t(7)=3.05, p<.02]$. Effects of attention were not significant with the other test gratings. The $0^{\circ}$ test grating perhaps exhibited a ceiling effect that obscured any effect of attention, and the $8^{\circ}$ test grating perhaps exhibited a floor effect that obscured any effect of attention. When we pooled that data across $2^{\circ}, 4^{\circ}$, and $6^{\circ}$, the effect of spatial attention on the TAE was again statistically reliable $(82 \%$ vs. $52 \%)[t(7)=2.65, p<.05]$.

At the smaller angles of tilt, the attended-side test gratings consistently produced a strong TAE. This was not so for the unattended side. Moreover, at $6^{\circ}$ of tilt, the attended-side test grating still exhibited a substantial TAE, whereas the unattended-side test grating no longer exhibited a TAE. These results provide a basic replication of the results of Experiment 1, under conditions in which central fixation was controlled by an eyetracker. It ap- pears that selective allocation of spatial attention modulates the occurrence and magnitude of the TAE.

\section{EXPERIMENT 3}

The following experiment was designed to test whether object-based attention, as opposed to spatial attention, can modulate the direct TAE. The participants viewed a symmetric plaid that comprised green bars oriented at $105^{\circ}$ and red bars oriented at $75^{\circ}$. During the viewing phase, the participants attended to either the green bars or the red bars. If the processing of orientation information were unaffected by the observer's selective allocation of object-based attention, the direct TAE from this symmetric plaid stimulus should be identical in green-attended and red-attended conditions (i.e., essentially zero).

\section{Method}

Participants. Twenty adults, all naive to the hypothesis, participated in this experiment. None of them were experienced psychophysical observers. Each participant participated in two trials.

Stimuli. The stimuli were presented on a 13-in. Macintosh color monitor. Cardboard over the screen revealed a circular aperture with a radius of $7.6^{\circ}$ of arc. The green grating in the plaid was oriented at $105^{\circ}$ and had a spatial frequency of 0.5 cycles/deg, 16\% luminance contrast, and a mean luminance of $4 \mathrm{~cd} / \mathrm{m}^{2}$. The red grating was oriented at $75^{\circ}$ and had a spatial frequency of 0.5 cycles $/ \mathrm{deg}$, $58 \%$ luminance contrast, and a mean luminance of $3 \mathrm{~cd} / \mathrm{m}^{2}$. A black fixation dot was placed in the center of the plaid, equidistant from the

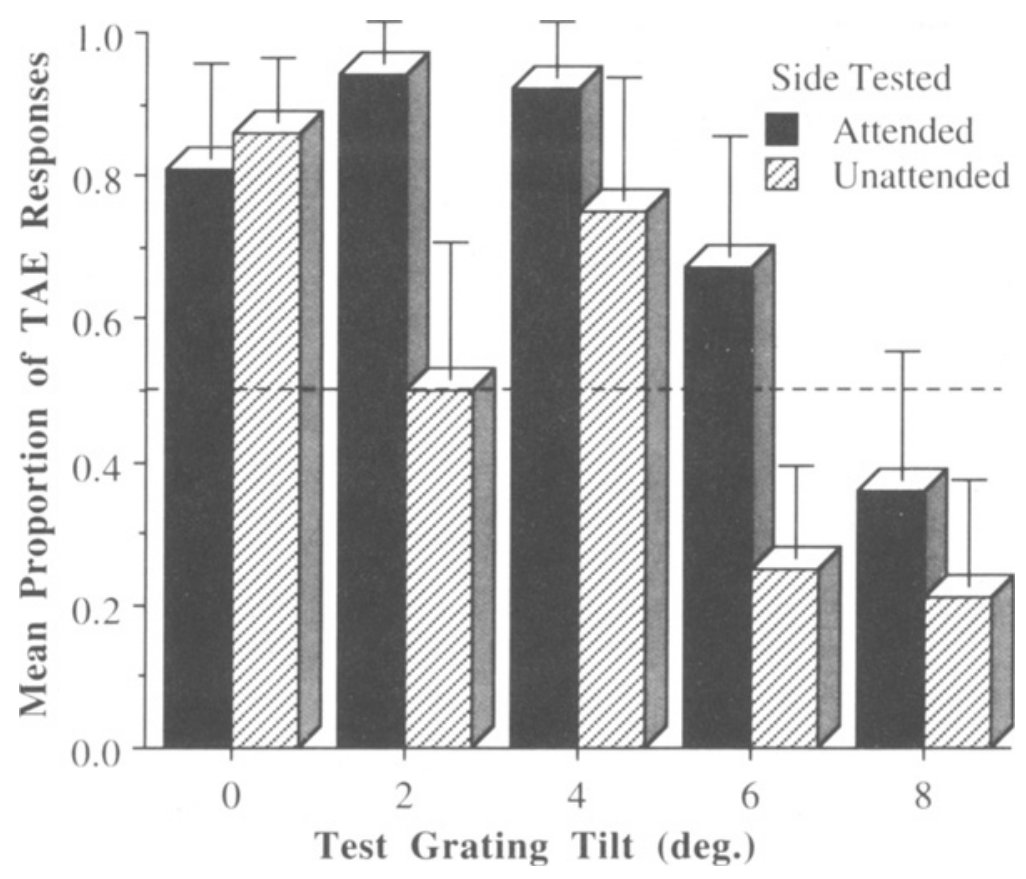

Figure 4. Experiment 2. Proportion of trials in which the briefly flashed test stimulus produced a TAE for the attended side and the unattended side. At $2^{\circ}$, the test grating was reliably perceived as on the other side of the vertical meridian when testing the attended side, but not when testing the unattended side. 
A
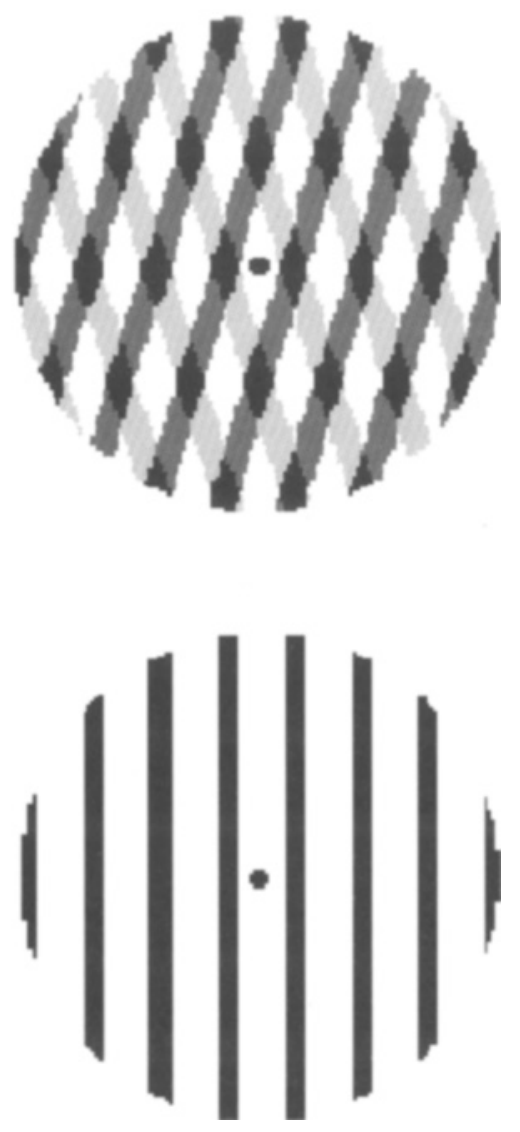

Figure 5. Participants viewed a symmetric plaid similar to that in panel $A$, in which the bars tilted counterclockwise were green and the bars tilted clockwise were red. During adaptation and fixation of the central dot, participants attended one of the colored gratings. After adaptation, participants viewed the grating in panel $B$ and judged the black bars as tilted slightly clockwise or counterclockwise.

green and red bars. The intersections of bars were black, and the background was white. The test grating was always at true vertical and contained a black fixation dot in its center. The test grating comprised black bars of the same spatial frequency as the other bars and had $97 \%$ luminance contrast and a mean luminance of $2.4 \mathrm{~cd} / \mathrm{m}^{2}$ (see Figure 5).

Procedure. The room lights were turned off during trials. The participants were instructed to fixate the center dot in the plaid and attend to one of the colors for $1 \mathrm{~min}$. The experimenter punctuated this adaptation period with reminders to fixate center and attend to the appropriate colored bars. After adaptation, the display was changed to the vertical test grating, and the participants were asked to maintain fixation on the center dot and to indicate whether the black bars appeared tilted slightly clockwise or counterclockwise. After a 2-min break, the second trial was run with the exact same stimuli, but this time the participant attended to the other colored grating. The order of which color was attended to first was counterbalanced across subjects.

\section{Results and Discussion}

Responses of clockwise were coded as $1 \mathrm{~s}$ and responses of counterclockwise were coded as $-1 \mathrm{~s}$. Occasionally, the participants refused to choose an alternative, insist- ing that the lines were indeed vertical. Those responses were coded as $0 \mathrm{~s}$. This coding system reflected the proportion of TAEs, from 0 to 1 , and their direction, positive as clockwise and negative as counterclockwise.

As is shown in Figure 6, when the participants attended to the green bars, the TAE was in the predicted direction (clockwise) with a proportion of 35 . When the participants attended to the red bars, the TAE was in the predicted direction (counterclockwise) with a proportion of .2. These different TAE proportions for the two attention conditions, elicited by identical stimuli, were significantly different from one another in a matched sample $t$ test $[t(19)=2.34, p<.05]$. Moreover, the TAE for the greenattended condition was significantly greater than 0 $[t(19)=2.33, p<.05]$. The TAE for the red-attended condition, however, was not reliably different from 0 $[t(19)=1.29, p>.1]$.

Since our coding of the "vertical" responses as 0 does not necessarily make the data normally distributed, an additional analysis was performed on only the trials in which a "clockwise" or "counterclockwise" response was given. When this binomial dataset was subjected to a chisquare analysis, the green-attended trials still showed reliably more "clockwise" responses $(9 / 11)$ than the redattended trials $(3 / 10)\left[\chi^{2}(1)=5.74, p<.02\right]$.

As with the previous experiments, there may still be some concern about the participants' not maintaining fixation on the central dot. If the participants tended to fixate along a nearby contour of the attended grating, instead of on the central dot, this might have blurred the other grating and reduced its effects. A control experiment was run to address this. Fourteen new naive participants viewed the same stimuli in two trials (order counterbalanced across subjects): one in which they were instructed to fixate along the nearby green contour during adaptation and one in which they were instructed to fixate along the nearby red contour during adaptation. The instructions made no reference to visual attention. As before, a few participants refused to choose one of the two forced alternatives and insisted that the test grating was perfectly vertical. Those responses were coded as $0 \mathrm{~s}$. In fact, when the participants did choose one of the alternatives (clockwise or counterclockwise), they often claimed to be making an arbitrary guess. In the green-contour-fixated condition, the participants averaged $.071 \mathrm{TAE}$ proportion, and in the red-contour-fixated condition, the participants averaged exactly 0 TAE proportion $[t(13)=.249, p>.5]$. The results of this control experiment suggest that the findings of Experiment 3 were not due to the participants' fixation strategies, but in fact were due to the participants' covertly attending to the individual colored gratings.

Although the overall proportion of TAEs in Experiment 3 was smaller than that with the briefly flashed stimuli of Experiment 2, the percentage of attentional modulation of the TAE in this experiment $(35 \%$ when attending green, $20 \%$ when attending red) is comparable to that observed in the previous experiments. Clearly, a TAE was present for this symmetric plaid stimulus when 


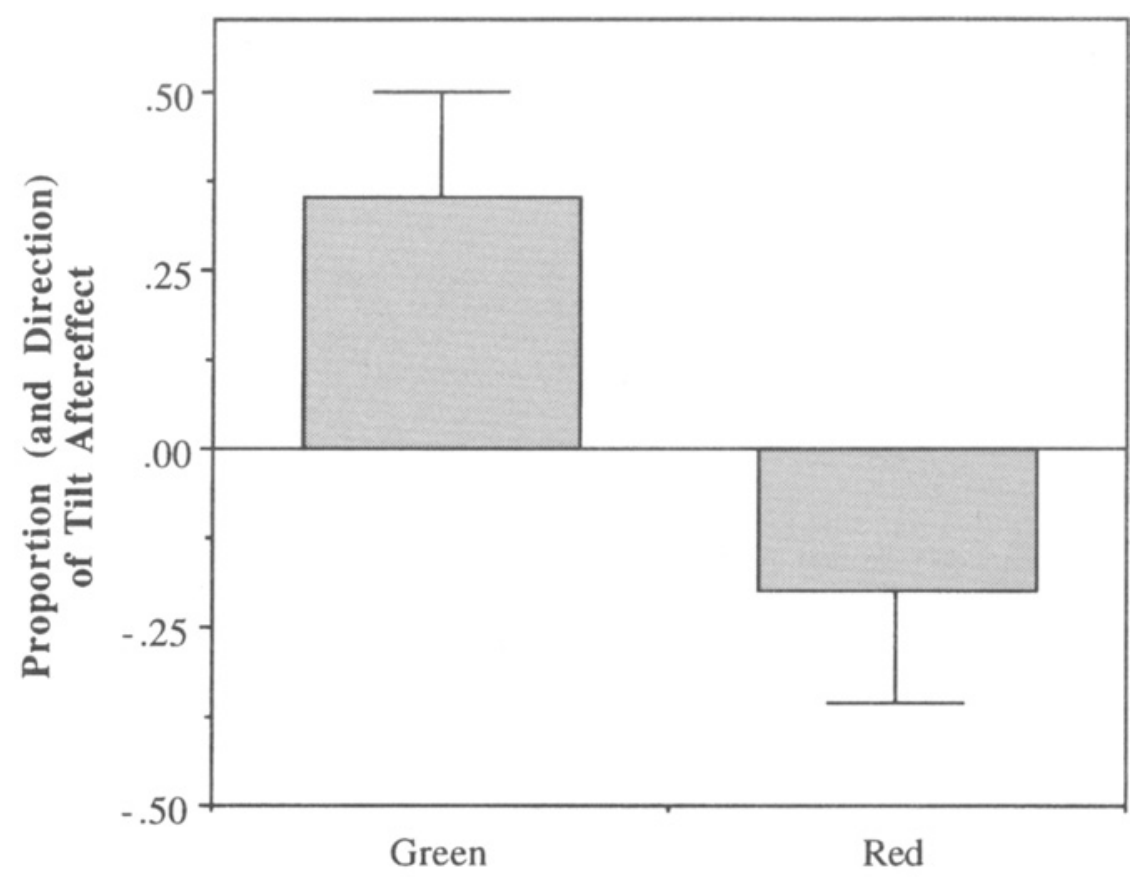

Attended Grating

\begin{abstract}
Figure 6. Experiment 3. The proportion of TAE in the test grating when participants had attended the green bars of the symmetric plaid compared with when they had attended the red bars of exactly the same plaid.
\end{abstract}

attention was allocated to one of its gratings (at least for the green grating). Such a result cannot easily be accounted for by a model in which the cortical locus of the TAE is immune to influences from voluntarily allocated object-based, or feature-based, visual attention.

\section{GENERAL DISCUSSION}

The present experiments tested whether selective visual attention modulates the magnitude and presence of the direct TAE. In Experiments $I$ and 2, attending to one of two identically oriented gratings, equidistant from fixation, increased the magnitude and occurrence of the TAE for that attended region relative to the unattended region. In Experiment 3, attending to one of two colored gratings in a symmetric plaid produced a TAE in the direction opposite the orientation of the attended grating. During adaptation to a tilted grating, the participant's attention toward a particular region (or object) of the visual scene produced a difference in the processing of orientation information associated with the attended and unattended regions (or objects). The direct TAE subsequently resulting from this orientation processing was thus greater for the attended region (or object) than for the unattended region (or object).

The mechanism(s) of spatial attention and object-based attention appear to impose an influence on the processing of orientation information at the level at which the direct TAE occurs, probably at the primary visual cortex (Blakemore et al., 1970; Blakemore \& Tobin, 1972; Carpenter \& Blakemore, 1973; Gilbert \& Weisel, 1983; Magnussen \& Kurtenbach, 1980; Morrone et al., 1982; Nelson \& Frost, 1978; Sillito, 1979; Wenderoth et al., 1989). Earlier results of visual attention's affecting perceptual aftereffects have avoided the claim of attention's having an early influence by proposing instead that the perceptual aftereffect in question has a later cortical locus. For example, a previous result of selective attention modulating the motion aftereffect was not used as evidence for attention modulating direction selectivity of complex cells in striate cortex (Chaudhuri, 1990). Instead, this attentional influence on the motion aftereffect was used as evidence for the motion aftereffect's being mediated partially by the extrastriate area MT, an area then believed to be more susceptible to attentional modulation (e.g., Corbetta, Miezen, Dobmeyer, Shulman, \& Petersen, 1990; Heinze et al., 1994; Moran \& Desimone, 1985).

However, functional interactions (feedforward, lateral, and feedback) among subsystems in the visual system are abundant (e.g., Churchland et al., 1994; Douglas et al., 1995; Duncan, 1996). In fact, striate cortex even has functional feedback projections to the lateral geniculate nucleus (Sillito, Jones, Gerstein, \& West, 1994). Thus, it is reasonable to expect any "top-down" attentional influence taking place in extrastriate regions to make its way down to striate cortex as well. Indeed, evi- 
dence of behaviorally relevant stimuli eliciting moderately greater responses from striate cells than those elicited by physically identical but behaviorally irrelevant stimuli indicates that attention might modulate the activity of cells in primary visual cortex of cats and monkeys (Artim \& Bridgeman, 1989; Haenny \& Schiller, 1988; Motter, 1993; Roelfsema et al., 1998). Finally, neuroimaging studies have shown modulation of metabolic activity in human primary visual cortex by visual attention (Brefczynski \& De Yoe, 1999; Gandhi et al., 1998; Sengpiel \& Hubener, 1999).

The present results of attention modulating the TAE contributes to this evidence that spatial attention, as well as object-based attention, influences activity in the primary visual cortex, since this cortical region appears to be the primary locus for orientation selectivity (DeValois, Yund, \& Hepler, 1982) and of the direct TAE (Wenderoth et al., 1989). However, this is not to say that adaptation of the sort that we have demonstrated here requires attention in order to take place. In Experiments 1 and 2, as well as in other studies (He, Cavanaugh, \& Intriligator, 1996; Houck \& Hoffman, 1986), the unattended stimulus still produced a detectable amount of adaptation and the resulting aftereffect.

Whether or not the locus of this attentional modulation involves striate cortex, it nonetheless appears to be an influence of voluntary selective attention on what is often assumed to be an automatic process-the TAE. These findings have important implications for cognitive theories of visual attention. First and foremost, they cast doubt on the idea that "preattentive" vision and "attentive" vision (cf. van der Heijden, 1996a, 1996b; Treisman, 1996) have independent architectural substrates. Moreover, these results corroborate evidence that selective attention can be allocated to objects and their properties as well as to locations (e.g., Bundesen, 1990; Humphreys, Olson, Romani, \& Riddoch, 1996; Vecera \& Farah, 1994; but see Braddick, 1990; Chaudhuri, 1990). Whether visual attention at a cognitive level is conceived of as a spotlight (Posner, Snyder, \& Davidson, 1980), a zoom lens (Eriksen \& St. James, 1986), a pointer (Ballard, Hayhoe, Pook, \& Rao, 1997), a set of spatial indices (Pylyshyn, $1989,1994)$, or as object files (Kahneman, Treisman, \& Gibbs, 1992), our results suggest that, at the level of visual feature extraction, visual attention can be realized as a change in activation of retinotopically organized orientation detectors - resulting in differential lateral inhibition and thus a modulation of the tilt aftereffect.

\section{REFERENCES}

Artim, J., \& Bridgeman, B. (1989). The physiology of attention: Participation of cat striate cortex in behavioral choice. Psychological Research, 50, 223-228.

Ballard, D. H., Hayhoe, M. M., Pook, P. K., \& RaO, R. P. N. (1997). Deictic codes for the embodiment of cognition. Behavioral \& Brain Sciences, 20, 723-767.

Bednar, J., \& MiIKKULAinen, R. (1997). A neural network model of visual tilt aftereffects. In Proceedings of the 19th Annual Conference of the Cognitive Science Society (pp. 37-42). Mahwah, NJ: Erlbaum.

Blakemore, C., Carpenter, R., \& Georgeson, M. (1970). Lateral inhibition between orientation detectors in the human visual system. Nature, 228, 37-39.

Blakemore, C., \& Tobin, E. A. (1972). Lateral inhibition between orientation detectors in the cat's visual cortex. Experimental Brain Research, 15, 439-440.

BradDICK, O. (1990). Switching off an after-effect. Nature, 344, 22

BREFCZYNSKI, J. A., \& DEYOE, E. A. (1999). A physiological correlate of the 'spotlight' of visual attention. Nature Neuroscience, 2, 370-374.

Broadbent, D. (1958). Perception and communication. London: Pergamon Press.

Bundesen, C. (1990). A theory of visual attention. Psychological Review, 97, 523-547.

CARPENTER, R., \& Blakemore, C. (1973). Interaction between orientations in human vision. Experimental Brain Research, 18, 287-303.

Chaudhurl, A. (1990). Modulation of the motion aftereffect by selective attention. Nature, 344, 60-62.

Churchland, P. S., Ramachandran, V., \& Sejnowski, T. (1994). A critique of pure vision. In C. Koch \& J. Davis (Eds.), Large-scale neuronal theories of the brain (pp. 23-60). Cambridge, MA: MIT Press.

Corbetta, M., Miezen, F., Dobmeyer, S., Shulman, G., \& PeterSEN, S. (1990). Attentional modulation of neural processing of shape, color, and velocity in humans. Science, 248, 1556-1559.

Deutsch, J., \& Deutsch, D. (1963). Attention: Some theoretical considerations. Psychological Review, 70, 80-90.

DeVAloIS, R. L., YUNd, E. W. \& HePLER, H. (1982). The orientation and direction selectivity of cells in macaque visual cortex. Vision Research, 22, 531-544.

Douglas, R. J., Koch, C., Mahowald, M., Martin, K.. \& Suarez. H. (1995). Recurrent excitation in neocortical circuits. Science, 269, 981-985.

DunCan, J. (1996). Cooperating brain systems in selective perception and action. In T. Inui \& J. McClelland (Eds.), Attention and performance XVI: Information integration in perception and communication (pp. 549-578). Cambridge, MA: MIT Press.

EBEnholTz, S. M. (1985). Absence of relational determination in the rod-and-frame effect. Perception \& Psychophysics, 37, 303-306.

ERIKSEN, C. W., \& ST. JAMES, J. D. (1986). Visual attention within and around the field of focal attention: A zoom lens model. Perception \& Psvchophysics, 40, 225-240.

Gandhi, S. P., Heeger, D. J., \& Boynton, G. M. (1998). Spatial attention affects brain activity in human primary visual cortex. Proceedings of the National Academy of Sciences, 96, 3314-3319.

GIBSON, J. J., \& RADNER, M. (1937). Adaptation, aftereffect and contrast in the perception of tilted lines. Journal of Experimental Psychology, 20, 453-467.

GILBERT, C. D. (1993). Circuitry, architecture, and functional dynamics of visual cortex. Cerebral Cortex, 3, 373-386.

GilberT, C. D., \& Weisel. T. N. (1983). Clustered intrinsic connections in cat visual cortex. Journal of Neuroscience, 3, 1116-1133.

HAENNY, P. E., \& SCHILLER, P. E. (1988). State dependent activity in monkey visual cortex: $I$. Single cell activity in V1 and V4 on visual tasks. Experimental Brain Research, 69, 225-244.

He, S., Cavanaugh, P., \& Intriligator, J. (1996). Attentional resolution and the locus of visual awareness. Nature, 383, 334-337.

heinze, H., Mangun, R., Burchert, W., Hinrichs, H., Scholz. M., Munte, T., Gos, A., SCherg, M.. Johannes, S.. Hundeshagen, H.. Gazzaniga, M., \& Hillyard, S. (1994). Combined spatial and temporal imaging of brain activity during visual selective attention in humans. Nature, 372, 543-556.

Houck, M. R., \& Hoffman, J. E. (1986). Conjunction of color and form without attention: Evidence from an orientation-contingent color aftereffect. Journal of Experimental Psychology: Human Perception \& Performance, 12, 186-199.

Humphreys, G. W., Olson, A., Romani, C., \& Riddoch, M. J. (1996). Competitive mechanisms of selection by space and object: A neuropsychological approach. In A. Kramer \& M. Coles (Eds.), Converg- 
ing operations in the study of visual selective attention (pp. 365-393). Washington, DC: American Psychological Association.

Kahneman. D. Treisman. A.. \& Gibbs. B. J. (1992). The reviewing of object files: Object-specific integration of information. Cognitive Psychology, 24, 175-219.

Lamme. V. A. F.. Super, H., \& Spekreisse, H. (1998). Feedforward, horizontal, and feedback processing in the visual cortex. Current Opinion in Neurobiology, 8, 529-535

LANKHEET. M. J.. \& Verstraten, F. (1995). Attentional modulation of adaptation to two-component transparent motion. Vision Research, $35,1401-1412$.

Livingstone. M., \& HuBEl., D. (1988). Segregation of form, color, movement and depth: Anatomy, physiology and perception. Science, 240, 740-750.

Magnussen. S. \& Kurtenbach, W. (1980). Adapting to two orientations: Disinhibition in a visual aftereffect. Science, 207, 908-909.

MARR, D. (1982). Vision. New York: Freeman.

MoRAn, J.. \& Desimone, R. (1985). Selective attention gates visual processing in the extrastriate cortex. Science, 229, 782-784.

Morant, R. B., \& Harris, J. R. (1965). Two different aftereffects of exposure to tilts. American Journal of Psvchology, 78, 218-226.

Morrone. M. C.. Burk. D. C.. \& MafFeI. L. ( 1982). Functional implications of cross-orientation inhibition of cortical visual cells: 1 . Neurophysiological evidence. Proceedings of the Roral Society of London: Series B, 216, 335-354.

MotTer. B. (1993). Focal attention produces spatially selective processing in visual cortical areas VI, V2, and V4 in the presence of competing stimuli. Jonunal of Neurophysiology, 70. 909-919.

MuIr. D.. \& Ovi:R. R. (1970). Tilt aftereffects in central and peripheral vision. Journal of Experimental Psychologi, 85, 165-170.

NELSON. J. I.. \& FROST. B. (1978). Orientation-selective inhibition from beyond the classic visual receptive field. Brain Research, 139, 359-365.

NORMAN. D. (1976). Memorr and attention (2nd ed.). New York: Wiley,

Posner. M. I.. Snyider. C. R.. \& Davidson. B. J. (1980). Attention and the detection of signals. Journal of Experimental Psychology: General, 109, 160-174.

PyLYSHYN. Z. W. (1989). The role of location indexes in spatial perception: A sketch of the FINST spatial-index model. Cognition, 32, 65-97.

Pyi.yshyn. Z. W. (1994). Some primitive mechanisms of spatial attention. Cognition. 50, 363-384.

Pyi.yshyn, Z. W. (1999). Is vision continuous with cognition? The case for cognitive impenetrability of visual perception. Behavioral \& Brain Sciences, 22, 341-423.

ReEs, G.. FrITH. C. \& L LAVIE. N. (1997). Modulating ir relevant motion perception by varying attentional load in an unrelated task. Science, 278, 1616-1619.

Roelfasma, P. R.. Lamme. V. A. F., \& Spekreijse. H. (1998). Objectbased attention in the primary visual cortex of the macaque monkey. Nature, 395, 376.

SEngitei. F.. \& Hubener. M. (1999). Visual attention: Spotlight on the primary visual cortex. Current Biolog!', 9. R318-R321

SHAi.l.1.: T. (1988). From neturopsycholog! to mental structure. Cambridge: Cambridge University Press.
Shulman, G. (1992). Attentional modulation of a figural aftereffect. Perception, 21, 7-19

Sil..iTo, A. M. (1979). Inhibitory mechanisms influencing complex cell orientation selectivity and their modification at high resting discharge levels. Journal of Physiology, 289, 33-53.

Sillito, A. M.. Jones, H. E., Gerstein, G. L.. \& West, D. C. (1994). Feature-linked synchronization of thalamic relay cell firing induced by feedback from the visual cortex. Nature, 369, 479-482.

SPIVEY-KNOWLTON, M. ( 1993). Simulating tilt illusions with lateral inhibition and a virtual axis. In Proceedings of the I5th Annual Conference of the Cognitive Science Society (pp. 953-958). Hillsdale. NJ: Erlbaum

TREISMAN. A. (1964). Effect of irrelevant material on the efficiency of selective listening. American Journat of Psvchology, 77, 533-546.

Treisman. A. (1996). Selection for perception for selection for action. Visual Cognition, 3, 353-357.

van der Heuden, A. H. C. (1996a). Two stages in visual information processing and visual perception? Visual Cognition, 3, 325-35.3.

van der Heuden, A. H. C. (1996b). Perception for selection, selection for action, and action for perception. Visual Cognition, 3, 357-361.

VeCERA, S. P., \& FARAH, M. J. (1994). Does visual attention select objects or locations? Journal of Experimental Psychology: General, 123. $146-160$

Wenieroth, P., VAN der ZWan, R., \& Johnstone, S. (1989). Mechanisms of orientation illusions. In D. Vickers \& P. Smith (Eds.), Human information processing: Measures, mechanisms and models (pp. 83106). North-Holland: Elsevier.

Wor.FE. J. ( 1984). Short test flashes produce large tilt aftereffects. Vision Research, 24, 1959-1964.

Yeh, S. L., Chen, I., DeVal ois, K.. \& DeValois. R. (1996). Figural aftereffects and visual attention. Journal of Experimental Psychology: Human Perception \& Performance, 22, 446-460.

ZEkI. S. (1993). A vision of the brain. Oxford: Blackwell.

\section{NOTE}

1. It is possible that after running one trial and making one judgment, the participants were biased on the second trial to make the opposite judgment, which might unfairly encourage supportive results for that second trial. To avoid this possibility, an independent samples $t$ test was performed on only the first judgments. In this half of the data, the greenattended condition showed a TAE proportion of .67 , and the redattended condition showed a TAE proportion of 0 ; again, they were reliably different from one another $[t(18)=2.25, p<.05]$. It is unclear why attending to the green bars would better facilitate a TAE than attending to the red bars. However, 3 participants (all of whom observed TAEs for the green-attended trial and not for the red-attended trial) reported that the green bars, which had a slightly lower luminance contrast, required "more effort" to attend.

(Manuscript received July 20, 1999; revision accepted for publication March 23, 2000.) 